\title{
New convolutions and their applicability to integral equations of Wiener-Hopf plus Hankel type
}

\author{
L. P. Castro*1 | R. C. Guerra ${ }^{1}$ | N. M. Tuan ${ }^{2}$
}

${ }^{1}$ Center for Research and Development in Mathematics and Applications (CIDMA), Department of Mathematics, University of Aveiro, Aveiro, Portugal

${ }^{2}$ Department of Mathematics, College of Education, Viet Nam National University, Hanoi, Viet Nam

†Accepted author’s manuscript (AAM) (New convolutions and their applicability to integral equations of Wiener-Hopf plus Hankel type, L. P. Castro, R. C. Guerra, N. M. Tuan, Mathematical Methods in the Applied Sciences, Volume 43, 4835-4846, Copyright (c) 2020 John Wiley \& Sons, Ltd., DOI: 10.1002/mma.6236). The final publication is available at https://doi.org/10.1002/mma.6236

Correspondence

*L. P. Castro. Email: castro@ua.pt

\begin{abstract}
Summary
We propose four new convolutions exhibiting convenient factorization properties associated with two finite interval integral transformations of Fourier-type together with their norm inequalities. Moreover, we study the solvability of a class of integral equations of Wiener-Hopf plus Hankel type (on finite intervals) with the help of the factorization identities of such convolutions. Fourier-type series are used to produce the solution formula of such equations and a Shannon-type sampling formula is also obtained.
\end{abstract}

\section{KEYWORDS:}

Convolution, integral equation, factorization, Fourier integral operator, Wiener-Hopf operator, Hankel operator.

\section{1 | INTRODUCTION AND PRELIMINARIES}

Introducing new convolutions has a direct impact in the areas of Integral Equations and Operator Theory, and different convolutions are as much important as many properties and applications they will be able to exhibit 12 [1/45]. Although the study of convolutions can also be seen as a classical subarea of Mathematical Analysis, the reason why it continues to attract the interest of several researchers is due to its high potential in exhibiting new properties and concrete applications. In fact, such convolutions can be seen as integral transforms that we can study from a theoretical point of view 6 (678. . Moreover, they can also be applied to problems modelled by certain integral equations, as we can see in different contexts ${ }^{9}$.

In the present paper, we will propose four new convolutions which will be written based on two parameters. Such parameters will enable these convolutions with extra levels of flexibility that are useful to enlarge the number of properties of their associated integral operators, as well as the number of possible applications (e.g., some classes of wave diffraction problems formulated as boundary value problems $\frac{10|11| 12|13| 14}{}$ can be analyzed and solved with the help of convolution operators of Wiener-Hopf plus Hankel type).

We will apply those new convolutions to the solvability analysis of a large class of integral equations. Such class of integral equations have brought about a great number of results in Mathematical Analysis, assembled in a huge number of works along the last decades $\$$ 15/2916/17]18/1920|21]. We are speaking about the class of Wiener-Hopf plus Hankel integral equations. Namely, in the present work, we will obtain the $L^{2}$-solution of a Wiener-Hopf plus Hankel equation, in terms of a Fourier-type series ${ }^{22}$. For 
that, we identify two finite integral transforms of Fourier-type which are associated with the set of our four new convolutions. The resulting realization will be the solution of that class of integral equations in terms of a Fourier-type series.

Then we will briefly present the main machinery that will be in use. We begin with the basic definition of the Fourier series.

Definition 1 (see $\left.e^{718}\right)$. Let $f$ be a Lebesgue integrable function on a finite interval $[0,2 \pi]$. The infinite sum

$$
\left(F_{f}\right)(x):=\frac{\hat{f}_{c}(0)}{2}+\sum_{n=1}^{\infty}\left[\hat{f}_{c}(n) \cos (n x)+\hat{f}_{s}(n) \sin (n x)\right]
$$

is called the Fourier series of $f$ on $[0,2 \pi]$, where $\hat{f}_{c}(n)$ and $\hat{f}_{s}(n)$ are the Fourier coefficients of $f$ defined by

$$
\begin{aligned}
& \hat{f}_{c}(n)=\frac{1}{\pi} \int_{0}^{2 \pi} \cos (n x) f(x) d x, n \in \mathbb{N}, \\
& \hat{f}_{s}(n)=\frac{1}{\pi} \int_{0}^{2 \pi} \sin (n x) f(x) d x, n \in \mathbb{N} .
\end{aligned}
$$

In the framework of this paper, the convergence is of the Lebesgue space $L^{2}([0,2 \pi])^{7 / 23 \mid 8}$. Let us write

$$
\mathscr{E}(x):=\frac{b \cos (x)+c \sin (x)}{\sqrt{\left(|b|^{2}+|c|^{2}\right) \pi}},
$$

with $b c \neq 0$. The series $(1)$ may be rewritten as follows

$$
\begin{aligned}
\left(F_{f}\right)(x) & =\frac{\hat{f}_{c}(0)}{2}+\sum_{n=1}^{\infty}\left\{\frac{\sqrt{\left(|b|^{2}+|c|^{2}\right) \pi}}{2}\left(\frac{1}{b} \hat{f}_{c}+\frac{1}{c} \hat{f}_{s}\right)(n) \mathscr{E}(n x)\right. \\
& \left.+\frac{\sqrt{\left(|b|^{2}+|c|^{2}\right) \pi}}{2}\left(\frac{1}{b} \hat{f}_{c}-\frac{1}{c} \hat{f}_{s}\right)(n) \mathscr{E}(-n x)\right\}=\frac{\hat{f}_{c}(0)}{2}+\sum_{0 \neq n=-\infty}^{+\infty} a_{n} \mathscr{E}(n x),
\end{aligned}
$$

where

$$
a_{n}=\left\{\begin{array}{lll}
\frac{\sqrt{\left(|b|^{2}+|c|^{2}\right) \pi}}{2}\left(\frac{1}{b} \hat{f}_{c}(n)-\frac{1}{c} \hat{f}_{s}\right)(n) & \text { if } & n<0 \\
\frac{\sqrt{\left(|b|^{2}+|c|^{2}\right) \pi}}{2 b} \hat{f}_{c}(n) & \text { if } & n=0 \\
\frac{\sqrt{\left(|b|^{2}+|c|^{2}\right) \pi}}{2}\left(\frac{1}{b} \hat{f}_{c}(n)+\frac{1}{c} \hat{f}_{s}\right)(n) & \text { if } & n>0 .
\end{array}\right.
$$

We will be using the usual inner product in $L^{2}([0,2 \pi])$. Namely, for any $f$ and $g$ in $L^{2}([0,2 \pi])$ their inner product is given by

$$
\langle f, g\rangle_{2}=\int_{0}^{2 \pi} f(x) \bar{g}(x) d x .
$$

The expression (5) leads us to the following result, which is also a consequence of the properties of the complete orthonormal basis $\left\{e^{i n x}\right\}_{n \in \mathbb{Z}}$ of the Hilbert space $L^{2}([0,2 \pi])$.

Proposition 1. The set of functions

is a complete orthonormal basis of $L^{2}([0,2 \pi])$.

$$
\left\{\frac{1}{\sqrt{2 \pi}}, \mathscr{E}(n x), n \in \mathbb{Z} \backslash\{0\}\right\}
$$

Proof. By (1) and (5), it is sufficient to prove the orthogonality of the system $\{\mathscr{E}(n x)\}_{n \in \mathbb{Z}}$. In view of this, we compute

$$
\begin{aligned}
\langle\mathscr{E}(n x), \mathscr{E}(m x)\rangle_{2} & =\frac{1}{\left(|b|^{2}+|c|^{2}\right) \pi} \int_{0}^{2 \pi}(b \cos (n x)+c \sin (n x))(\bar{b} \cos (m x)+\bar{c} \sin (m x)) d x \\
& = \begin{cases}0, & n \neq m \\
1, & n=m,\end{cases}
\end{aligned}
$$

for $n, m \neq 0$. 
For $n=m=0$, we have

$$
\left\langle\frac{1}{\sqrt{2 \pi}}, \frac{1}{\sqrt{2 \pi}}\right\rangle_{2}=\frac{1}{2 \pi} \int_{0}^{2 \pi} 1 d x=1 .
$$

For $n=0$ and $m \neq 0$, we have

$$
\left\langle\frac{1}{\sqrt{2 \pi}}, \mathscr{E}(m x)\right\rangle_{2}=\frac{1}{\pi \sqrt{2\left(|b|^{2}+|c|^{2}\right)}} \int_{0}^{2 \pi}(\bar{b} \cos (m x)+\bar{c} \sin (m x)) d x=0 .
$$

In what follows, the notation $\left(F_{f}\right)(x)$ of the Fourier series will be replaced by $\mathcal{E}_{f}(x)$ given by (5). Furthermore, the representation in the form (5) naturally suggests us to define a finite Fourier-type transformation as follows.

Definition 2 (Finite two parameters Fourier-type transformation). (a) Let $f$ be a Lebesgue integrable function on [0,2 $\pi$ ]. The finite (two parameters) Fourier-type transformation of $f$ corresponding to $\mathscr{E}(n x)$ is defined by

$$
\left(T_{1} f\right)(n):=\frac{1}{2} \sqrt{\frac{|b|^{2}+|c|^{2}}{\pi}} \int_{0}^{2 \pi}\left(\frac{1}{b} \cos (n x)+\frac{1}{c} \sin (n x)\right) f(x) d x, n \in \mathbb{Z} .
$$

(b) The infinite sum

$$
\left(\mathcal{E}_{f}\right)(x):=\sum_{n \in \mathbb{Z}}\left(T_{1} f\right)(n) \mathscr{E}(n x)
$$

is called finite Fourier-type series of $f$ on $[0,2 \pi]$ corresponding to $\mathscr{E}(n x)$.

We observe that the constant before the integral sign in $(9)$ is chosen for later purposes.

Let us recall that

$$
\ell_{2}:=\left\{\left\{s_{n}\right\}_{n}: s_{n} \in \mathbb{C} \text { with } \sum_{n \in \mathbb{Z}}\left|s_{n}\right|^{2}<\infty\right\},
$$

stands for the Hilbert space of all square summable complex series.

Let us also recall the known Riesz-Fischer theorem, which states that a measurable function on $[-\pi, \pi]$ is square integrable if and only if the corresponding Fourier series converges (in the space $\left.L^{2}\right)$. In other words, for any $f \in L^{2}([-\pi, \pi])$ and its $n$-th Fourier coefficient given by

$$
F_{n}=\frac{1}{2 \pi} \int_{-\pi}^{\pi} f(x) e^{-i n x} d x \quad(n \in \mathbb{Z})
$$

we have

$$
f(x)=\sum_{n \in \mathbb{Z}} F_{n} e^{i n x} \quad \text { for almost every } x \in(-\pi, \pi) ;
$$

conversely, for any $\left\{a_{n}\right\}_{n=-\infty}^{n=+\infty} \in \ell_{2}$, there exists a function $f \in L^{2}([-\pi, \pi])$ such that the values $a_{n}$ are the Fourier coefficients of $f$. In view of this, the integral transform (11) defines an invertible bounded linear operator between two Hilbert spaces $L^{2}([-\pi, \pi])$ and $\ell_{2}$ in which the inversion formula is given by (12).

Remark 1. The above consideration of $T_{1}$ is natural for the extension $[10)$, as $\frac{1}{b} \cos (n x)+\frac{1}{c} \sin (n x)$ is the inversion kernel of the basis $b \cos (n x)+c \sin (n x)=\sqrt{\left(|b|^{2}+|c|^{2}\right) \pi} \mathscr{E}(n x)$.

Put

$$
\begin{aligned}
T_{1}: L^{2}([0,2 \pi]) & \rightarrow \ell_{2} \\
f & \mapsto\left\{T_{1} f\right\}_{n \in \mathbb{Z}} .
\end{aligned}
$$

By (5), (9), and (10) we can state the following theorem.

Theorem 1. The integral transform (9) defines a bounded linear map from $L^{2}(-\pi, \pi)$ to $\ell_{2}$ whose inversion map is given by (10).

The following propositions are immediate consequences of the finite Fourier integral transformation case. 
Proposition 2 (Uniqueness theorem). If $f \in L^{1}([0,2 \pi])$, with $\left(T_{1} f\right)(n)=0$, for all $n \in \mathbb{Z}$, then $f \equiv 0$ (in $L^{1}$-norm).

Proposition 3 (Riemann-Lebesgue lemma). If $f \in L^{1}([0,2 \pi])$, then

$$
\lim _{n \rightarrow \infty}\left(T_{1} f\right)(n)=0
$$

We will also consider

$$
\left(T_{2} f\right)(n):=\frac{1}{2} \sqrt{\frac{|b|^{2}+|c|^{2}}{\pi}} \int_{0}^{2 \pi}\left(\frac{1}{b} \cos (n x)-\frac{1}{c} \sin (n x)\right) f(x) d x, n \in \mathbb{Z} .
$$

Since $T_{2}(n)=T_{1}(-n)$ for every $n \in \mathbb{Z}$, the above propositions also obviously work for the integral transform $T_{2}$. Due to the circumstance that $T_{2}(n)=T_{1}(-n)$, we can also say that $T_{2}$ corresponds to the composition of $T_{1}$ with the reflection operator in frequency domain.

\section{2 | FOUR NEW CONVOLUTIONS AND NORM INEQUALITIES}

In this section, we construct four new generalized convolutions associated with $T_{1}$ and $T_{2}$, and prove some norm inequalities.

Definition 3. For any two functions $f, g$ that are Lebesgue integrable on $[0,2 \pi]$, and where $f$ is a function defined on $\mathbb{R}$ and $2 \pi$-periodic, we define four (two parameter) finite Fourier-type convolutions as follows:

$$
\begin{aligned}
& \left(f \underset{T_{1}, T_{1}, T_{1}}{*} g\right)(x):=\frac{1}{8 b c^{2}} \sqrt{\frac{|b|^{2}+|c|^{2}}{\pi}} \int_{0}^{2 \pi}\left[\left(3 c^{2}-b^{2}\right) f(x-v)+\left(b^{2}+c^{2}\right) f(x+v)\right) \\
& \left.+\left(b^{2}+c^{2}\right) f(-x+v)-\left(b^{2}+c^{2}\right) f(-x-v)\right] g(v) d v ; \\
& \left(f_{T_{1}, T_{1}, T_{2}}^{*} g\right)(x):=\frac{1}{8 b c^{2}} \sqrt{\frac{|b|^{2}+|c|^{2}}{\pi}} \int_{0}^{2 \pi}\left[\left(b^{2}+c^{2}\right) f(x-v)+\left(3 c^{2}-b^{2}\right) f(x+v)\right. \\
& \left(f \begin{array}{c}
\left.f_{T_{1}, T_{2}, T_{1}}^{*} g\right)(x):=\frac{1}{8 b c^{2}} \sqrt{\frac{|b|^{2}+|c|^{2}}{\pi}} \int_{0}^{2 \pi}\left[\left(b^{2}+c^{2}\right) f(x-v)-\left(b^{2}+c^{2}\right) f(x+v)\right. \\
\left(f \underset{T_{1}, T_{2}, T_{2}}{*} g\right)(x):=\frac{1}{8 b c^{2}} \sqrt{\frac{|b|^{2}+|c|^{2}}{\pi}} \int_{0}^{2 \pi}\left[-\left(3 c^{2}-b^{2}\right) f(-x+v)+\left(b^{2}+c^{2}\right) f(-x-v)\right] g(v) d v ; g(x-v) d v ;\left(b^{2}+c^{2}\right) f(x+v) \\
\left.+\left(b^{2}+c^{2}\right) f(-x+v)+\left(3 c^{2}-b^{2}\right) f(-x-v)\right] g(v) d v .
\end{array}\right.
\end{aligned}
$$

The next theorem clarifies the relation of those convolutions with the previously considered operators.

Theorem 2 (Convolution theorem). If $f, g$ are Lebesgue integrable on $[0,2 \pi]$ and $f$ is a function defined on $\mathbb{R}$ and $2 \pi$-periodic, then each one of the convolutions introduced in Definition 3 has two factorization identities associated with the integral operators $T_{1}$ and $T_{2}$, and the corresponding norm inequality:

$$
\begin{aligned}
& \left(T_{j}\left(f_{T_{1}, T_{1}, T_{1}}^{*} g\right)\right)(n)=\left(T_{j} f\right)(n)\left(T_{j} g\right)(n), \text { for } n \in \mathbb{Z}, \\
& \left\|f \underset{T_{1}, T_{1}, T_{1}}{*} g\right\|_{1} \leq \frac{2|b|^{2}+3|c|^{2}}{4|b||c|^{2}} \sqrt{\frac{|b|^{2}+|c|^{2}}{\pi}}\|f\|_{1}\|g\|_{1} ; \\
& \left(T_{j}\left(f_{T_{1}, T_{1}, T_{2}}^{*} g\right)\right)(n)=\left(T_{j} f\right)(x)\left(T_{k} g\right)(n), \text { for } n \in \mathbb{Z} \text {, } \\
& \left\|f_{T_{1}, T_{1}, T_{2}}^{*} g\right\|_{1} \leq \frac{2|b|^{2}+3|c|^{2}}{4|b||c|^{2}} \sqrt{\frac{|b|^{2}+|c|^{2}}{\pi}}\|f\|_{1}\|g\|_{1} ; \\
& \left(T_{j}\left(f_{T_{1}, T_{2}, T_{1}}^{*} g\right)\right)(n)=\left(T_{k} f\right)(x)\left(T_{j} g\right)(n), \text { for } n \in \mathbb{Z} \text {, }
\end{aligned}
$$




$$
\begin{aligned}
& \left\|f \underset{T_{1}, T_{2}, T_{1}}{*} g\right\|_{1} \leq \frac{2|b|^{2}+3|c|^{2}}{4|b||c|^{2}} \sqrt{\frac{|b|^{2}+|c|^{2}}{\pi}}\|f\|_{1}\|g\|_{1} ; \\
& \left(T_{j}\left(f_{T_{1}, T_{2}, T_{2}}^{*} g\right)\right)(n)=\left(T_{k} f\right)(x)\left(T_{k} g\right)(n), \text { for } n \in \mathbb{Z} \text {, } \\
& \left\|f_{T_{1}, T_{2}, T_{2}}^{*} g\right\|_{1} \leq \frac{2|b|^{2}+3|c|^{2}}{4|b||c|^{2}} \sqrt{\frac{|b|^{2}+|c|^{2}}{\pi}}\|f\|_{1}\|g\|_{1},
\end{aligned}
$$

with $j=1$ and $k=2$, or $j=2$ and $k=1$.

Proof. We first prove the factorization identity (17). In this case, the factorization identity just includes one of the operators, $T_{1}$ or $T_{2}$, and we will prove it for $j=1$, this is, for the operator $T_{1}$. So, considering the kernel of the operator $T_{1}$, we can rewrite the product of two kernels of this type as a linear combination of four terms of the same type in suitable variables, as follows

$$
\begin{aligned}
& {\left[\frac{1}{b} \cos (n u)+\frac{1}{c} \sin (n u)\right]\left[\frac{1}{b} \cos (n v)+\frac{1}{c} \sin (n v)\right]} \\
& \quad=\frac{1}{4 b c^{2}}\left\{\left(3 c^{2}-b^{2}\right)\left[\frac{1}{b} \cos (n(u+v))+\frac{1}{c} \sin (n(u+v))\right]\right. \\
& +\left(b^{2}+c^{2}\right)\left[\frac{1}{b} \cos (n(u-v))+\frac{1}{c} \sin (n(u-v))\right] \\
& +\left(b^{2}+c^{2}\right)\left[\frac{1}{b} \cos (n(-u+v))+\frac{1}{c} \sin (n(-u+v))\right] \\
& \left.-\left(b^{2}+c^{2}\right)\left[\frac{1}{b} \cos (n(-u-v))+\frac{1}{c} \sin (n(-u-v))\right]\right\} .
\end{aligned}
$$

Using this identity, changing appropriately the variables and noting the $2 \pi$-periodicity of $f$, we have

$$
\begin{aligned}
\left(T_{1} f\right)(n)\left(T_{1} g\right)(n)= & \frac{|b|^{2}+|c|^{2}}{4 \pi} \int_{0}^{2 \pi} \int_{0}^{2 \pi}\left[\frac{1}{b} \cos (n u)+\frac{1}{c} \sin (n u)\right] \\
= & \frac{|b|^{2}+|c|^{2}}{4 b c^{2}(4 \pi)} \int_{0}^{2 \pi} \int_{0}^{2 \pi}\left\{\left(3 c^{2}-b^{2}\right)\left[\frac{1}{b} \cos (n(u+v))+\frac{1}{c} \sin (n(u+v))\right]\right. \\
& +\left(b^{2}+c^{2}\right)\left[\frac{1}{b} \cos (n(u-v))+\frac{1}{c} \sin (n(u-v))\right] \\
& +\left(b^{2}+c^{2}\right)\left[\frac{1}{b} \cos (n(-u+v))+\frac{1}{c} \sin (n(-u+v))\right] f(u) g(v) d u d v \\
& \left.-\left(b^{2}+c^{2}\right)\left[\frac{1}{b} \cos (n(-u-v))+\frac{1}{c} \sin (n(-u-v))\right]\right\} f(u) g(v) d u d v \\
= & \frac{|b|^{2}+|c|^{2}}{4 b c^{2}(4 \pi)} \int_{0}^{2 \pi} g(v)\left\{\int_{v}^{2 \pi+v}\left(3 c^{2}-b^{2}\right)\left[\frac{1}{b} \cos (n x)+\frac{1}{c} \sin (n x)\right] f(x-v) d x\right. \\
& +\int_{-v}^{2 \pi-v}\left(b^{2}+c^{2}\right)\left[\frac{1}{b} \cos (n x)+\frac{1}{c} \sin (n x)\right] f(x+v) d x \\
& -\int_{v}^{-2 \pi+v}\left(b^{2}+c^{2}\right)\left[\frac{1}{b} \cos (n x)+\frac{1}{c} \sin (n x)\right] f(-x+v) d x \\
& \left.+\int_{-v}^{-2 \pi-v}\left(b^{2}+c^{2}\right)\left[\frac{1}{b} \cos (n x)+\frac{1}{c} \sin (n x)\right] f(-x-v) d x\right\} d v \\
&
\end{aligned}
$$




$$
\begin{aligned}
& =\frac{|b|^{2}+|c|^{2}}{4 b c^{2}(4 \pi)} \int_{0}^{2 \pi} g(v)\left\{\int_{v}^{2 \pi+v}\left(3 c^{2}-b^{2}\right)\left[\frac{1}{b} \cos (n x)+\frac{1}{c} \sin (n x)\right] f(x-v) d x\right. \\
& +\int_{-v}^{2 \pi-v}\left(b^{2}+c^{2}\right)\left[\frac{1}{b} \cos (n x)+\frac{1}{c} \sin (n x)\right] f(x+v) d x \\
& +\int_{-2 \pi+v}^{v}\left(b^{2}+c^{2}\right)\left[\frac{1}{b} \cos (n x)+\frac{1}{c} \sin (n x)\right] f(-x+v) d x \\
& \left.-\int_{-2 \pi-v}^{-v}\left(b^{2}+c^{2}\right)\left[\frac{1}{b} \cos (n x)+\frac{1}{c} \sin (n x)\right] f(-x-v) d x\right\} d v \\
& =\frac{1}{2} \sqrt{\frac{|b|^{2}+|c|^{2}}{\pi}} \int_{0}^{2 \pi}\left[\frac{1}{b} \cos (n x)+\frac{1}{c} \sin (n x)\right] \\
& \left\{\frac { 1 } { 8 b c ^ { 2 } } \sqrt { \frac { | b | ^ { 2 } + | c | ^ { 2 } } { \pi } } \int _ { 0 } ^ { 2 \pi } \left[\left(3 c^{2}-b^{2}\right) f(x-v)+\left(b^{2}+c^{2}\right) f(x+v)\right.\right. \\
& \left.\left.+\left(b^{2}+c^{2}\right) f(-x+v)-\left(b^{2}+c^{2}\right) f(-x-v)\right] g(v) d v\right\} d x \\
& =T_{1}\left(f_{T_{1}, T_{1}, T_{1}}^{*} g\right)(n) .
\end{aligned}
$$

Now, let us prove the corresponding norm inequality

$$
\begin{aligned}
\int_{0}^{2 \pi}\left|\left(f \underset{T_{1}, T_{1}, T_{1}}{*} g\right)(x)\right| d x \leq & \frac{1}{8|b||c|^{2}} \sqrt{\frac{|b|^{2}+|c|^{2}}{\pi}} \int_{0}^{2 \pi} \int_{0}^{2 \pi}\left[\left(3|c|^{2}+|b|^{2}\right)|f(x-v)|\right. \\
& +\left(|b|^{2}+|c|^{2}\right)|f(x+v)|+\left(|b|^{2}+|c|^{2}\right)|f(-x+v)| \\
& \left.+\left(|b|^{2}+|c|^{2}\right)|f(-x-v)|\right]|g(v)| d v d x \\
= & \frac{4|b|^{2}+6|c|^{2}}{8|b||c|^{2}} \sqrt{\frac{|b|^{2}+|c|^{2}}{\pi}} \int_{0}^{2 \pi} \int_{0}^{2 \pi}|f(y)||g(u)| d v d y \\
= & \frac{2|b|^{2}+3|c|^{2}}{4|b||c|^{2}} \sqrt{\frac{|b|^{2}+|c|^{2}}{\pi}}\|f\|_{1}\|g\|_{1} .
\end{aligned}
$$

We will omit the proofs for the cases of the other convolutions, because they are similar to this one, using, of course, the corresponding different identities. The following identities help us to obtain the factorizations for the other convolutions, when we consider $j=1$ and $k=2$, through the decomposition presented for the factorization (17). Namely, we have:

$$
\begin{aligned}
& {\left[\frac{1}{b} \cos (n u)+\frac{1}{c} \sin (n u)\right]\left[\frac{1}{b} \cos (n v)-\frac{1}{c} \sin (n v)\right]} \\
& =\left[\frac{1}{b} \cos (n u)+\frac{1}{c} \sin (n u)\right]\left[\frac{1}{b} \cos (-n v)+\frac{1}{c} \sin (-n v)\right] ; \\
& {\left[\frac{1}{b} \cos (n u)-\frac{1}{c} \sin (n u)\right]\left[\frac{1}{b} \cos (n v)+\frac{1}{c} \sin (n v)\right]} \\
& =\left[\frac{1}{b} \cos (-n u)+\frac{1}{c} \sin (-n u)\right]\left[\frac{1}{b} \cos (n v)+\frac{1}{c} \sin (n v)\right] ; \\
& {\left[\frac{1}{b} \cos (n u)-\frac{1}{c} \sin (n u)\right]\left[\frac{1}{b} \cos (n v)-\frac{1}{c} \sin (n v)\right]} \\
& =\left[\frac{1}{b} \cos (-n u)+\frac{1}{c} \sin (-n u)\right]\left[\frac{1}{b} \cos (-n v)+\frac{1}{c} \sin (-n v)\right] .
\end{aligned}
$$


We will also omit the proofs of the factorization identities when we consider $j=2$ and $k=1$, since they are very similar to the proofs of the factorization identities with $j=1$ and $k=2$. In fact, after proving the factorization identity for the first convolution, with $j=2$, we can use the following identities to obtain the corresponding decompositions associated with the other convolutions, when considering $j=2$ and $k=1$. Namely, we have:

$$
\begin{aligned}
& {\left[\frac{1}{b} \cos (n u)-\frac{1}{c} \sin (n u)\right] } {\left[\frac{1}{b} \cos (n v)+\frac{1}{c} \sin (n v)\right] } \\
&= {\left[\frac{1}{b} \cos (n u)-\frac{1}{c} \sin (n u)\right]\left[\frac{1}{b} \cos (n v)-\frac{1}{c} \sin (-n v)\right] ; } \\
& {\left[\frac{1}{b} \cos (n u)+\frac{1}{c} \sin (n u)\right]\left[\frac{1}{b} \cos (n v)-\frac{1}{c} \sin (n v)\right] } \\
&=\left[\frac{1}{b} \cos (n u)-\frac{1}{c} \sin (-n u)\right]\left[\frac{1}{b} \cos (n v)-\frac{1}{c} \sin (n v)\right] \\
& {\left[\frac{1}{b} \cos (n u)+\frac{1}{c} \sin (n u)\right] } \\
&=\left[\frac{1}{b} \cos (n v)+\frac{1}{c} \sin (n v)\right] \\
&=\left[\frac{1}{b} \cos (n u)-\frac{1}{c} \sin (-n u)\right]\left[\frac{1}{b} \cos (n v)-\frac{1}{c} \sin (-n v)\right] .
\end{aligned}
$$

Remark 2. Looking at the four convolutions, we see that:

(i) If $b^{2}+c^{2}=0$, for instance $b=1$ and $c=i, T_{1}$ and $T_{2}$ become the finite Fourier and inverse Fourier transformations and all the constructed convolutions are unified in the unique well-known Fourier convolution.

(ii) If $3 c^{2}-b^{2}=b^{2}+c^{2}$ or, equivalently, $b= \pm c$, then the considered transforms are exactly the finite Hartley transformations, and all the mentioned convolutions turn out the recently published Hartley convolutions ${ }^{2 / 24}$.

(iii) If $3 c^{2}-b^{2}=0$, each convolution has just three terms. For instance, if $b=\sqrt{3}$ and $c=1$ then $T_{1}$ and $T_{2}$ are symmetric (Hermitian) transformations, and the convolution (13) yields

$$
\left(f \underset{T_{1}, T_{1}, T_{1}}{*} g\right)(x):=\frac{1}{\sqrt{3 \pi}} \int_{0}^{2 \pi}[f(x+v)+f(-x+v)-f(-x-v)] g(v) d v .
$$

For convenience, let us write $\tilde{f}(t):=f(-t)$ which corresponds to the reflection of the function $f$ in time domain. It is surprising that the convolution (25) does not contain the so-called convolution term. Actually, it is constituted by three terms: two cross-correlation terms, that are $f(x+v)$ and $\widetilde{f}(x+v)$, together with the convolution term of the reflection $\tilde{f}$, that is $f(-x+v)$. The form of this convolution is notably different from the previously published convolutions. Of course, the three other convolutions (14), (15), and (16) have the usual convolution term $f(x-v)$. From our point of view, the convolution (25) may be used for processing reflective periodic signals in practical problems.

In summary, the well-known Fourier and Hartley convolutions, and the convolution given by (25) can be considered as special cases among the studied family of convolutions, in some sense.

As we will see in Section 3 the factorization identities in Theorem 2 are essential for solving Fredholm integral equations with mixed kernels. Before going to Section 3 we should confirm the $L^{2}$-boundedness of the constructed convolution type operators.

Due to the fact of $L^{2}([0,2 \pi]) \subset L^{1}([0,2 \pi])$, we can state the following theorem.

Theorem 3. Let $f$ be a function defined on $\mathbb{R}$ and $2 \pi$-periodic. If $f, g \in L^{2}([0,2 \pi])$, then the following norm inequality holds

$$
\|f \underset{T}{*} g\|_{2} \leq \frac{\sqrt{2\left(|b|^{2}+|c|^{2}\right)\left(\left|3 c^{2}-b^{2}\right|^{2}+3\left|b^{2}+c^{2}\right|^{2}\right)}}{4|b||c|^{2}}\|f\|_{2}\|g\|_{2},
$$

where, for short, the notation ${ }_{T}^{*}$ represents any of the convolution type operators $13-16$. 
Proof. We will just prove the theorem for the convolution (13), since the other ones can be proceeded in a similar way. By the Cauchy-Schwarz inequality, we have $|a+b+c+d|^{2} \leq 4\left(|a|^{2}+|b|^{2}+|c|^{2}+|d|^{2}\right)$ and so,

$$
\begin{aligned}
\left|\left(f \underset{T_{1}, T_{1}, T_{1}}{*} g\right)(x)\right|^{2}= & \left|\frac{1}{8 b c^{2}} \sqrt{\frac{|b|^{2}+|c|^{2}}{\pi}}\right|^{2} \mid \int_{0}^{2 \pi}\left[\left(3 c^{2}-b^{2}\right) f(x-v)+\left(b^{2}+c^{2}\right) f(x+v)\right. \\
& \left.+\left(b^{2}+c^{2}\right) f(-x+v)-\left(b^{2}+c^{2}\right) f(-x-v)\right]\left.g(v) d v\right|^{2} \\
\leq & \frac{|b|^{2}+|c|^{2}}{64|b|^{2}|c|^{4} \pi} \int_{0}^{2 \pi}|g(v)|^{2} d v \int_{0}^{2 \pi} \mid\left(3 c^{2}-b^{2}\right) f(x-v)+\left(b^{2}+c^{2}\right) f(x+v) \\
& +\left(b^{2}+c^{2}\right) f(-x+v)-\left.\left(b^{2}+c^{2}\right) f(-x-v)\right|^{2} d v \\
\leq & \frac{|b|^{2}+|c|^{2}}{64|b|^{2}|c|^{4} \pi} \int_{0}^{2 \pi}|g(v)|^{2} d v \int_{0}^{2 \pi} 4\left[\left|3 c^{2}-b^{2}\right|^{2}|f(x-v)|^{2}+\left|b^{2}+c^{2}\right|^{2}|f(x+v)|^{2}\right. \\
& \left.+\left|b^{2}+c^{2}\right|^{2}|f(-x+v)|^{2}+\left|b^{2}+c^{2}\right|^{2}|f(-x-v)|^{2}\right] d v \\
\leq & \frac{4\left(|b|^{2}+|c|^{2}\right)\left(\left|3 c^{2}-b^{2}\right|^{2}+3\left|b^{2}+c^{2}\right|^{2}\right)}{64|b|^{2}|c|^{4} \pi}\|f\|_{2}^{2}\|g\|_{2}^{2} .
\end{aligned}
$$

This implies that

$$
\left\|f \underset{T_{1}, T_{1}, T_{1}}{*} g\right\|_{2}=\left(\int_{0}^{2 \pi}\left|\left(f \underset{T_{1}, T_{1}, T_{1}}{*} g\right)(x)\right|^{2} d x\right)^{\frac{1}{2}} \leq \frac{\sqrt{2\left(|b|^{2}+|c|^{2}\right)\left(\left|3 c^{2}-b^{2}\right|^{2}+3\left|b^{2}+c^{2}\right|^{2}\right)}}{4|b||c|^{2}}\|f\|_{2}\|g\|_{2},
$$

which proves the proposition.

\section{3 | ON THE SOLVABILITY OF WIENER-HOPF PLUS HANKEL EQUATIONS}

In this section, we will study the solvability of the following Fredholm integral equation

$$
\lambda \varphi(x)+\int_{0}^{2 \pi} K(x, u) \varphi(u) d u=f(x)
$$

where $\varphi$ is unknown, $\lambda \in \mathbb{C}, K(x, y):=p(x-u)+q(x+u)$, with $p, q$ and $f$ being given functions in which $p$ and $q$ are $2 \pi$-periodic one-variable functions.

We will use the above introduced convolutions to analyze the solvability of this equation, and to obtain the explicit solution in the form of a Fourier-type series.

We start by introducing some auxiliary functions. Let

$$
\begin{aligned}
A(n) & :=\lambda+\frac{1}{2 b} \sqrt{\frac{\pi}{|b|^{2}+|c|^{2}}}\left[\left(3 b^{2}-c^{2}\right)\left(T_{1} p\right)(n)+\left(b^{2}+c^{2}\right)\left(T_{2} p\right)(n)+\left(b^{2}+c^{2}\right)\left(T_{1} q\right)(n)\right. \\
& \left.-\left(b^{2}+c^{2}\right)\left(T_{2} q\right)(n)\right] ; \\
B(n) & :=\frac{1}{2 b} \sqrt{\frac{\pi}{|b|^{2}+|c|^{2}}}\left[\left(b^{2}+c^{2}\right)\left(T_{1} p\right)(n)-\left(b^{2}+c^{2}\right)\left(T_{2} p\right)(n)+\left(3 b^{2}-c^{2}\right)\left(T_{1} q\right)(n)\right. \\
& \left.+\left(b^{2}+c^{2}\right)\left(T_{2} q\right)(n)\right] ; \\
D(n) & :=A(n) A(-n)-B(n) B(-n) ; \\
D_{1}(n) & :=A(-n)\left(T_{1} f\right)(n)-B(n)\left(T_{2} f\right)(n) ; \\
D_{2}(n) & :=A(n)\left(T_{2} f\right)(n)-B(-n)\left(T_{1} f\right)(n) .
\end{aligned}
$$


Theorem 4. Assume that the functions $p$ and $q$ are $2 \pi$-periodic. Moreover, assume that they are piecewise continuous and $f \in L^{2}([0,2 \pi])$.

(i) If $\lambda \neq 0$, then there exists an integer $K^{*}$ such that $D(n) \neq 0$, for every $n \geq K^{*}$.

(ii) If $D(n) \neq 0$, for every $n \in \mathbb{N}$, then equation 27 , has a unique solution for every $f \in L^{2}([0,2 \pi])$, which is given by

$$
\varphi(x)=\sum_{n \in \mathbb{Z}} \frac{D_{1}(n)}{D(n)} \mathscr{E}(n x) .
$$

Proof. (i) By the Riemann-Lebesgue lemma for $T_{1}$ and $T_{2}$, we deduce that

$$
\lim _{n \rightarrow \infty} D(n)=\lambda^{2} \neq 0 .
$$

Hence, there exists an integer $K^{*} \in \mathbb{N}$ such that $D(n) \neq 0$, for all $n \geq K^{*}$.

(ii) The coefficients $3 c^{2}-b^{2}, b^{2}+c^{2}, b^{2}+c^{2},-\left(b^{2}+c^{2}\right)$ that appear in the right-hand sides of the convolutions (13)-(16) constitute a symmetric matrix as

$$
M:=\left(\begin{array}{cccc}
3 c^{2}-b^{2} & b^{2}+c^{2} & b^{2}+c^{2} & -\left(b^{2}+c^{2}\right) \\
b^{2}+c^{2} & 3 c^{2}-b^{2} & -\left(b^{2}+c^{2}\right) & b^{2}+c^{2} \\
b^{2}+c^{2} & -\left(b^{2}+c^{2}\right) & 3 c^{2}-b^{2} & b^{2}+c^{2} \\
-\left(b^{2}+c^{2}\right) & b^{2}+c^{2} & b^{2}+c^{2} & 3 c^{2}-b^{2}
\end{array}\right),
$$

with $\operatorname{det}(M)=-256 b^{2} c^{6} \neq 0$, since $b c \neq 0$ as assumed above.

Therefore, replacing $g$ with $\varphi$ and $f$ with $p$ in (13)-(16) and converting those expressions (the fact of $\operatorname{det}(M)=-256 b^{2} c^{6} \neq 0$ allows these conversions), we obtain

$$
\begin{aligned}
\int_{0}^{2 \pi} p(x-u) \varphi(u) d u= & \frac{1}{2 b} \sqrt{\frac{\pi}{|b|^{2}+|c|^{2}}}\left[\left(3 b^{2}-c^{2}\right)\left(p_{T_{1}}^{*} \varphi\right)(x)+\left(b^{2}+c^{2}\right)\left(p_{T_{1}, T_{1}, T_{2}}^{*} \varphi\right)(x)\right. \\
& +\left(b^{2}+c^{2}\right)\left(p_{T_{1}, T_{2}, T_{1}}^{*} \varphi\right)(x)-\left(b^{2}+c^{2}\right)\left(p_{T_{1}, T_{2}, T_{2}}^{*} \underset{*}{*} \varphi(x)\right] .
\end{aligned}
$$

Again, replacing $g$ with $\varphi$ and $f$ with $q$ and converting those expressions as above, we get

$$
\begin{aligned}
& \int_{0}^{2 \pi} q(x+u) \varphi(u) d u=\frac{1}{2 b} \sqrt{\frac{\pi}{|b|^{2}+|c|^{2}}}\left[\left(b^{2}+c^{2}\right)\left(q_{T_{1}}^{*} \varphi\right)(x)+\left(3 b^{2}-c^{2}\right)\left(q_{T_{1}, T_{1}, T_{2}}^{*} \varphi\right)(x)\right. \\
& \left.-\left(b^{2}+c^{2}\right)\left(q \underset{T_{1}, T_{2}, T_{1}}{*} \varphi\right)(x)+\left(b^{2}+c^{2}\right)\left(q \underset{T_{1}, T_{2}, T_{2}}{*} \varphi\right)(x)\right] .
\end{aligned}
$$

Applying $T_{1}$ to both sides of these identities and using the factorization identities of the convolutions appeared on the right-hand side, we obtain

$$
\begin{aligned}
& T_{1}\left(\int_{0}^{2 \pi} p(x-u) \varphi(u) d u\right)(n)=\frac{1}{2 b} \sqrt{\frac{\pi}{|b|^{2}+|c|^{2}}}\left[\left(3 b^{2}-c^{2}\right)\left(T_{1} p\right)(n)\left(T_{1} \varphi\right)(n)\right. \\
& \left.\quad+\left(b^{2}+c^{2}\right)\left(T_{1} p\right)(n)\left(T_{2} \varphi\right)(n)+\left(b^{2}+c^{2}\right)\left(T_{2} p\right)(n)\left(T_{1} \varphi\right)(n)-\left(b^{2}+c^{2}\right)\left(T_{2} p\right)(n)\left(T_{2} \varphi\right)(n)\right] ; \\
& T_{1}\left(\int_{0}^{2 \pi} q(x+u) \varphi(u) d u\right)(n)=\frac{1}{2 b} \sqrt{\frac{\pi}{|b|^{2}+|c|^{2}}}\left[\left(b^{2}+c^{2}\right)\left(T_{1} q\right)(n)\left(T_{1} \varphi\right)(n)\right. \\
& \left.\quad+\left(3 b^{2}-c^{2}\right)\left(T_{1} q\right)(n)\left(T_{2} \varphi\right)(n)-\left(b^{2}+c^{2}\right)\left(T_{2} q\right)(n)\left(T_{1} \varphi\right)(n)+\left(b^{2}+c^{2}\right)\left(T_{2} q\right)(n)\left(T_{2} \varphi\right)(n)\right] .
\end{aligned}
$$

Analogously, applying the operator $T_{2}$ as above, we have

$$
\begin{aligned}
& T_{2}\left(\int_{0}^{2 \pi} p(x-u) \varphi(u) d u\right)(n)=\frac{1}{2 b} \sqrt{\frac{\pi}{|b|^{2}+|c|^{2}}}\left[\left(3 b^{2}-c^{2}\right)\left(T_{2} p\right)(n)\left(T_{2} \varphi\right)(n)\right. \\
& \left.\quad+\left(b^{2}+c^{2}\right)\left(T_{2} p\right)(n)\left(T_{1} \varphi\right)(n)+\left(b^{2}+c^{2}\right)\left(T_{1} p\right)(n)\left(T_{2} \varphi\right)(n)-\left(b^{2}+c^{2}\right)\left(T_{1} p\right)(n)\left(T_{1} \varphi\right)(n)\right] ;
\end{aligned}
$$




$$
\begin{aligned}
& T_{2}\left(\int_{0}^{2 \pi} q(x+u) \varphi(u) d u\right)(n)=\frac{1}{2 b} \sqrt{\frac{\pi}{|b|^{2}+|c|^{2}}}\left[\left(b^{2}+c^{2}\right)\left(T_{2} q\right)(n)\left(T_{2} \varphi\right)(n)\right. \\
& \left.\quad+\left(3 b^{2}-c^{2}\right)\left(T_{2} q\right)(n)\left(T_{1} \varphi\right)(n)-\left(b^{2}+c^{2}\right)\left(T_{1} q\right)(n)\left(T_{2} \varphi\right)(n)+\left(b^{2}+c^{2}\right)\left(T_{1} q\right)(n)\left(T_{1} \varphi\right)(n)\right] .
\end{aligned}
$$

To prove the uniqueness of the solution of equation (27), we invoke the Fredholm alternative theorem. Suppose that the homogeneous equation corresponding to equation 27 , has a solution $\varphi_{*} \in L^{2}([0,2 \pi])$, this is,

$$
\lambda \varphi_{*}(x)+\int_{0}^{2 \pi}[p(x-u)+q(x+u)] \varphi_{*}(u) d u=0 .
$$

Applying $T_{1}$ and $T_{2}$ to both sides of this equation and using identities $35-(38)$, we obtain the following system of two linear equations

$$
\left\{\begin{array}{l}
A(n)\left(T_{1} \varphi_{*}\right)(n)+B(n)\left(T_{2} \varphi_{*}\right)(n)=0 \\
B(-n)\left(T_{1} \varphi_{*}\right)(n)+A(-n)\left(T_{2} \varphi_{*}\right)(n)=0 .
\end{array}\right.
$$

Since $D(n) \neq 0$, for every $n \in \mathbb{N}_{0}$, we obtain $\left(T_{1} \varphi_{*}\right)(n)=\left(T_{2} \varphi_{*}\right)(n)=0$, for $n \geq 0$. Due to the "uniqueness theorems" of the operators $T_{1}$ and $T_{2}$, we obtain that $\varphi_{*}=0$. Thus, the homogeneous equation associated with the equation (27) has only the trivial solution and so, by the Fredholm alternative theorem, equation 27) has a unique non-trivial solution, provided $0 \neq f \in L^{2}([0,2 \pi])$.

Now, we will obtain the solution formula 33 . Suppose that $\varphi \in L^{2}([0,2 \pi])$ is fulfilling $[27$. Proceeding in a similar way as used for the homogeneous equation, we obtain the following system

$$
\left\{\begin{array}{l}
A(n)\left(T_{1} \varphi\right)(n)+B(n)\left(T_{2} \varphi\right)(n)=\left(T_{1} f\right)(n) \\
B(-n)\left(T_{1} \varphi\right)(n)+A(-n)\left(T_{2} \varphi\right)(n)=\left(T_{2} f\right)(n) .
\end{array}\right.
$$

Since $D(n) \neq 0$ for every $n \geq 0$, this system has a unique solution given by

$$
\left(T_{1} \varphi\right)(n)=\frac{D_{1}(n)}{D(n)}, \quad\left(T_{2} \varphi\right)(n)=\frac{D_{2}(n)}{D(n)}=\frac{D_{1}(-n)}{D(n)}=\left(T_{1} \varphi\right)(-n),
$$

for $n=0,1, \ldots$ Taking the inverse transform of $T_{1}$, we obtain

$$
\varphi(x)=\sum_{n \in \mathbb{Z}} \frac{D_{1}(n)}{D(n)} \mathscr{E}(n x),
$$

which belongs to $L^{2}([0,2 \pi])$ and fulfills 27 for almost every $x \in[0,2 \pi]$, where

$$
\|\varphi\|_{2}^{2}=\frac{\pi}{2}\left|\hat{f}_{c}(0)\right|^{2}+\sum_{0 \neq n \in \mathbb{Z}}\left|\frac{D_{1}(n)}{D(n)}\right|^{2}<\infty .
$$

Remark 3. The convolution functions given by (13), [14), [15, and (16) are linearly independent. In other words, each one of those four convolution functions cannot be expressed as a linear combination of the three others. This fact is an immediate consequence of $\operatorname{det}(M) \neq 0$ as shown by (34).

Remark 4. (i) It is known that $T_{1}, T_{2}$ and their inverses, $T_{1}^{-1}$ and $T_{2}^{-1}$, are integral transformations from the time domain to frequency domain, and vice versa, respectively. Looking at the solution $\varphi$ given by [33), we can state that the formula can be seen as a new version of the Shannon sampling theorem for periodic signals sampled in the frequency domain, in which we can recover all signal frequencies from the knowledge of the information at the frequency samples $\frac{D_{1}(n)}{D(n)}$. In practice, the above formula has some advantage when computing data directly in the frequency domain, instead of that in the time domain. 
(ii) Rewriting the function $D$ in terms of the finite cosine and sine Fourier transformations, considering

$$
\begin{aligned}
& \left(\hat{f}_{c}\right)(n)=\frac{1}{\pi} \int_{0}^{2 \pi} \cos (n x) f(x) d x ; \\
& \left(\hat{f}_{s}\right)(n)=\frac{1}{\pi} \int_{0}^{2 \pi} \sin (n x) f(x) d x,
\end{aligned}
$$

we obtain

$$
D(n)=\lambda^{2}+2 \lambda \pi\left(\hat{p}_{c}\right)(n)+\pi^{2}\left[\left(\hat{p}_{c}\right)^{2}(n)-\left(\hat{q}_{c}\right)^{2}(n)+\left(\hat{p}_{s}\right)^{2}(n)-\left(\hat{q}_{s}\right)^{2}(n)\right] .
$$

Having this form of $D$ in mind, we can obtain the following proposition, whose proof is direct, just assuming that $D(n) \neq 0$ and solving the equation in terms of $\lambda$.

We end up by describing a criterion for $D(n) \neq 0$ (emphasizing, by this way, that proposition (ii) in Theorem 4 is only a sufficient condition).

Proposition 4. Suppose that the functions $p$ and $q$ in the equation $(27)$ are Lebesgue integrable. The determinant $D(n) \neq 0$ if and only if

$$
\lambda \neq \pi\left[-\left(\hat{p}_{c}\right)(n) \pm \sqrt{\left(\hat{q}_{c}\right)^{2}(n)+\left(\hat{q}_{s}\right)^{2}(n)-\left(\hat{p}_{s}\right)^{2}(n)}\right]
$$

\section{ACKNOWLEDGMENTS}

This work was supported in part by FCT-Portuguese Foundation for Science and Technology through the Center for Research and Development in Mathematics and Applications (CIDMA) of Universidade de Aveiro, within project UIDB/04106/2020, and by the Viet Nam National Foundation for Science and Technology Development (NAFOSTED). R. C. Guerra also acknowledges the direct support of the Portuguese Foundation for Science and Technology (FCT) through the scholarship PD/BD/114187/2016.

\section{Conflict of interest}

The authors declare that there are no conflicts of interest to this work.

\section{References}

1. Anh PK, Castro LP, Thao PT, Tuan NM. Two new convolutions for the fractional Fourier transform. Wireless Pers Commun. 2017;92(2):623-637.

2. Anh PK, Tuan N M, Tuan PD. The finite Hartley new convolutions and solvability of the integral equations with Toeplitz plus Hankel kernels. J Math Anal Appl. 2013;397(2):537-549.

3. Castro LP, Guerra RC, Tuan NM. On Wiener's Tauberian theorems and convolution for oscillatory integral operators. Turkish J Math. 2019;43(3):1124-1147.

4. Castro LP, Minh LT, Tuan NM. New convolutions for quadratic-phase Fourier integral operators and their applications. Mediterr J Math. 2018;15(1):17pp.

5. Castro LP, Saitoh S. New convolutions and norm inequalities. Math Ineq Appl. 2012;15(3):707-716.

6. Bogveradze G, Castro LP. Toeplitz plus Hankel operators with infinite index. Integral Equations Operator Theory. 2008;62(1):43-63.

7. Debnath L, Bhatta D. Integral Transforms and Their Applications. Boca Raton: CRC Press; 2015. 3rd ed. 
8. Stein EM, Shakarchi R. Fourier Analysis: An Introduction. In: Princeton Lectures in Analysis. Princeton: Princeton University Press 2003. Volume 1.

9. Castro LP, Guerra RC, Tuan NM. New convolutions weighted by Hermite functions and their applications. Math Ineq Appl. 2019;22(2):719-745.

10. Castro LP, Kapanadze D. Dirichlet-Neumann-impedance boundary value problems arising in rectangular wedge diffraction problems. Proc. Amer. Math. Soc.. 2008;136(6):2113-2123.

11. Castro LP, Kapanadze D. Wave diffraction by a half-plane with an obstacle perpendicular to the boundary. J. Differential Equations. 2013;254(2):493-510.

12. Castro LP, Kapanadze D. Mixed boundary value problems of diffraction by a half-plane with an obstacle perpendicular to the boundary. Math. Methods Appl. Sci.. 2014;37(10):1412-1427.

13. Castro LP, Speck F-O, Teixeira FS. On a class of wedge diffraction problems posted by Erhard Meister. Oper. Theory Adv. Appl.. 2004;147:213-240.

14. Castro LP, Speck F-O, Teixeira FS. Mixed boundary value problems for the Helmholtz equation in a quadrant. Integral Equations Operator Theory. 2006;56(1):1-44.

15. Anderson BDO, Kailath T. Fast algorithms for the integral equations of the inverse scattering problem. Integral Equations Operator Theory. 1978;1:132-136.

16. Castro LP, Itou H, Saitoh S, Tuan NM, Tuan PD. Numerical solutions of linear singular integral equations by means of Tikhonov regularization and reproducing kernels. Anal Appl (Singap). 2012;38(4):1261-1276.

17. Castro LP, Rojas EM. Explicit solutions of Cauchy singular integral equations with weighted Carleman shift. J Math Anal Appl. 2010;371(1):128-133.

18. Castro LP, Rojas EM, Saitoh S, Tuan NM, Tuan PD. Solvability of singular integral equations with rotations and degenerate kernels in the vanishing coefficient case. Anal Appl (Singap). 2015;13(1):1-21.

19. Chadan K, Sabatier PC. Inverse Problems in Quantum Scattering Theory. New York: Springer-Verlag; 1977.

20. Kailath T, Levy B, Ljung L, Morf M. Fast time-invariant implementations of Gaussian signal detectors. IEEE Trans. Information Theory. 1978;24(4):469-477.

21. Tsitsiklis JN, Levy BC. Integral Equations and Resolvents of Toeplitzplus Hankel Kernels. Technical Report LIDS-P-1170: Laboratory for Information and Decision Systems, M.I.T.; 1981.

22. Zygmund A. Trigonometric Series. Cambridge: Cambridge University Press; 2002. Volumes I \& II combined.

23. Katznelson Y. An Introduction to Harmonic Analysis. Cambridge: Cambridge University Press; 2004.3 rd ed.

24. Bracewell RN. The Hartley Transform. Oxford: Oxford University Press; 1986. 